\title{
THE MICROWAVE BACKGROUND RADIATION
}

\author{
G. De Zotti, L. Danese, L. Toffolatti, and A. Franceschini \\ Astronomical Observatory and Department of Astronomy \\ Vicolo dell' Osservatorio, 5 \\ I-35122 Padova, Italy
}

\begin{abstract}
We review the data on the spectrum and isotropy of the microwave background radiation and the astrophysical processes that may produce spectral distortions and anisotropies. As yet no fully satisfactory explanation has been found for the submillimeter excess observed by Matsumoto et al. (1988). The most precise data at $\lambda>1 \mathrm{~mm}$ disagree with nonrelativistic comptonization models which match the excess. Distortions produced by a very hot intergalactic medium yielding the $\mathrm{X}$-ray background do not fit the submillimeter data. Very special requirements must be met for the interpretation in terms of high-redshift dust emission to work.

Reported anisotropies on scales of several degrees and of tens of arcsec may be produced, at least in part, by discrete sources. Because the best experiments at $\mathrm{cm}$ wavelengths are close to the confusion limit, they provide interesting information on the large-scale distribution of radio sources.
\end{abstract}

\section{MEASUREMENTS OF THE SPECTRUM AND THEIR IMPLICATIONS}

As demonstrated by Table 1, our knowledge of the microwave background radiation (MWBR) spectrum has substantially improved in the last few years. (We have omitted the results by Peterson, Richards and Timusk [1985] because systematic errors have recently been discovered in them [Richards 1989]). Earlier measurements have been discussed by Weiss (1980), who carried out an exhaustive analysis of the observational problems. For reviews of theoretical work on spectral distortions, see Danese and De Zotti (1977), Sunyaev and Zeldovich (1980), De Zotti (1986), and Bond (1988).

TABLE 1. Recent Measurements of the MWBR Brightness Temperature

\begin{tabular}{|c|c|c|}
\hline References & $\lambda(\mathrm{cm})$ & $T_{M W B}(K)$ \\
\hline Sironi et al. 1989 & 50.0 & $3.0 \pm 1.0$ \\
\hline Levin et al. 1988 & 1.3 & $2.11 \pm 0.38$ \\
\hline Sironi and Bonelli 1986 & 2.0 & $2.79 \pm 0.15$ \\
\hline De Amici et al. 1988 & 8.1 & $2.59 \pm 0.13$ \\
\hline De Amici et al. 1989 & 7.9 & $2.63 \pm 0.11$ \\
\hline Mandolesi et al. $1986 \mathrm{~b}$ & 6.3 & $2.70 \pm 0.07$ \\
\hline Kogut et al. 1988 & 3.0 & $2.61 \pm 0.06$ \\
\hline Johnson and Wilkinson 1987 & 1.2 & $2.783 \pm 0.025$ \\
\hline De Amici et al. 1985 & 0.909 & $2.81 \pm 0.12$ \\
\hline Bersanelli et al. 1989 & 0.333 & $2.60 \pm 0.09$ \\
\hline Meyer and Jura 1985 & 0.264 & $2.70 \pm 0.04$ \\
\hline Meyer, Roth, and Hawkins 1989 & 0.264 & $2.76 \pm 0.07$ \\
\hline Crane et al. 1989 & 0.264 & $2.796_{-0.039}^{+0.014}$ \\
\hline Meyer, Roth, and Hawkins 1989 & 0.132 & $2.83 \pm 0.09$ \\
\hline Crane et al. 1989 & 0.132 & $2.83 \pm 0.07$ \\
\hline Matsumoto et al. 1988 & 0.116 & $2.799 \pm 0.018$ \\
\hline Matsumoto et al. 1988 & 0.0709 & $2.955 \pm 0.017$ \\
\hline Matsumoto et al. 1988 & 0.0481 & $3.175 \pm 0.027$ \\
\hline Matsumoto et al. 1988 & 0.0262 & $<4.105$ \\
\hline
\end{tabular}




\subsection{The Submillimeter Excess}

The most spectacular recent result is the detection (Matsumoto et al. 1988) of a large excess in the Wien region, qualitatively similar to that reported earlier by Gush (1981), comprising $\approx 10 \%$ of the total energy of the MWBR. The upper limits set by Bernstein $e$ t al. (1989) on intermediate to large-scale fluctuations of this excess sharply constrain the possibility of a local origin. On the assumption that it is real and nonlocal, various possible explanations have been proposed and discussed (Hayakawa et al. 1987; Carr 1988; Smoot et al. 1988; Bond 1988; Bond, Carr and Hogan 1989; De Zotti and Toffolatti 1989).

1.1.1. Comptonization by primeval plasma. A distortion of this kind was predicted by Field and Perrenod (1977) as associated with a hot intergalactic plasma yielding the 2-50 keV X-ray background. The best fit to the data listed in Table 1 corresponds to $T_{0}=2.82 \mathrm{~K}$ and to a comptonization parameter $y=(2.02 \pm 0.16) \times 10^{-2}$, remarkably close to Field and Perrenod's prediction $\left(y \simeq 1.5 \times 10^{-2}\right)$. The fit, however, is not good, mainly because there is an apparent conflict between the two submillimeter data points, favouring an even larger value for $y$, and the two most accurate results at $\lambda>1 \mathrm{~mm}$ (Johnson and Wilkinson 1987; Crane et al. 1989), which are consistent with $y=0$. Also, the energy requirements are daunting, and exotic mechanisms might be needed (Lacey and Field 1988; Field and Walker 1989; Wang and Field, this volume).

As first pointed out by Wright (1979), however, the original calculation of Field and Perrenod was not entirely self-consistent, since nonrelativistic formulae were used even though most of the X-ray emission came from mildly relativistic electrons. A correct calculation, following Fabbri (1981), yields a substantially worse fit. The more refined models, worked out by Guilbert and Fabian (1986), Barcons (1987), and Barcons and Fabian (1988), also produce an unacceptable fit, generally because the predicted MWBR flux at $709 \mu \mathrm{m}$ and $481 \mu \mathrm{m}$ is below that reported by Matsumoto et al. (1988), while the upper limit at $262 \mu \mathrm{m}$ tends to be exceeded. We conclude that a hot intergalactic gas cannot account for both the X-ray background and the submillimeter excess reported by Matsumoto et al. (see also Rogers and Field 1989; Taylor and Wright 1989).

1.1.2. High redshift dust. Bond, Carr and Hogan (1986), Negroponte (1986) and McDowell (1986) showed that dust at high redshifts would emit in the far-infrared (far-IR), with a peak at $\lambda \approx 700 \mu \mathrm{m}$, i.e., almost precisely where the peak of the excess of Matsumoto $e t$ al. lies. On the other hand, as shown by Lacey and Field (1988), stars formed with the same mass function as in the solar neighborhood cannot supply the required energy; other sources such as very massive objects, accreting black holes, or decaying particles are needed. Also, it is extremely difficult to generate enough dust at sufficiently high redshifts both in the framework of scaleinvariant biased cold dark matter models and of pancake models; hierarchical models with more power at small scales could, however, do the job (Bond, Carr and Hogan 1989).

In any case, very special conditions must be met in order to account for the detailed shape of the observed excess. For example, under the usual assumption of a dust absorption efficiency approximately constant at the effective frequencies of the heating radiation, and proportional to $\lambda^{-\alpha}$, with $\alpha \approx 2$, at far-IR wavelengths, the temperature of a grain of size $r_{g}$ heated by a radiation field of intensity $I$, is roughly proportional to $\left(I / r_{g}\right)^{1 / 6}$. Even if the intensity of the radiation field is strictly uniform, the standard grain size distribution, ranging from $50 \AA$ to $2500 \AA$, translates into a temperature distribution having a width of at least $(2500 / 50)^{1 / 6}=2$. A further smearing out is expected, because of inhomogeneities in the radiation field intensity and because of the redshift distribution of the emitting dust.

In contrast, after subtraction of a blackbody at $T=2.755 \pm 0.017 \mathrm{~K}$ (the weighted mean of data listed in Table 1, excluding those by Matsumoto et al. [1988]) we end up with a residual spectrum well fitted by a single dust temperature $\left[T_{d}=3.58 \pm 0.24 \mathrm{~K}\right.$ for $\left.\alpha=2\right]$, and substantially 
narrower than the dust emission spectra observed in a variety of astrophysical settings (De Zotti and Toffolatti 1989).

1.1.3. Decaying particles. Kawasaki and Sato (1987) claimed that a good fit may be obtained with comptonization of photons produced by radiative decay of light, weakly interacting particles having appropriate mass, lifetime, and number density. Although their analysis was not entirely self-consistent (De Zotti and Toffolatti 1989), their general conclusion stands, only with somewhat different values of the relevant parameters (Burigana 1989). On the other hand, Raffelt, Dearborn and Silk (1989) showed that the full range of masses and lifetimes that could account for the submillimeter excess is excluded by consideration of the associated energy losses from stars.

1.1.4. Tests for the submillimeter excess. Two methods have been proposed to obtain a consistency check on spectral distortions and, at the same time, to distinguish between local and cosmological contributions. Both exploit differential observations, bypassing the difficulties related to absolute calibrations.

A first possibility is provided by measurements of the dipole anisotropy: in the presence of the reported rise of the MWBR brightness temperature, the Compton-Getting effect would lead to a decrease of the dipole amplitude at submillimeter wavelengths (Danese and De Zotti 1981; Lubin 1982). This expectation is not borne out by the measurements of Halpern et al. (1988), although the disagreement is only at the $2 \sigma$ level (also, the direction of their dipole is somewhat different: it may be that emission from the Galaxy or some bright submillimeter source has contaminated the data). The effect is proportional to the first derivative of the radiation brightness and is, consequently, sensitive to the detailed shape of the distortion; it could therefore also help to discriminate between different models.

Another test relies on the frequency dependence of the Sunyaev-Zeldovich (1972) effect (Gould and Rephaeli 1978; Fabbri and Melchiorri 1979; Rephaeli 1980; Wright 1983). The change in the MWBR brightness due to scattering of photons by hot $\left(k T_{e} \gg h v\right)$, but nonrelativistic, electrons in rich clusters of galaxies is proportional to the second derivative of the radiation brightness and, therefore, emphasizes sharp features in the spectrum.

\subsection{The Spectrum at $\lambda>1 \mathrm{~mm}$}

A key factor in improving the accuracy of the results in the range $10 \mathrm{~cm}$ to a few $\mathrm{mm}$ has been a careful investigation of the atmospheric emission (Danese and Partridge 1989, and references therein). As a bonus, these investigations also led to a better understanding of the atmospheric emission itself.

There is still room for significant improvements, particularly longward of $\lambda \simeq 10 \mathrm{~cm}$, where Bose-Einstein-like and bremsstrahlung distortions are expected to show up. A big problem here is the accurate modelling of the emission from our own Galaxy (cf. Sironi et al. 1989). The essentially isotropic component due to unresolved discrete sources can be estimated by exploiting the very deep source counts, now available at several radio frequencies (De Zotti and Toffolatti 1989).

Even when the results of Matsumoto et al. (1988) are ignored, the fit to an unperturbed blackbody spectrum is not fully satisfactory. We find $\chi_{\min }^{2}=18.5$ for 11 degrees of freedom, for a best-fit blackbody temperature $T_{B B}=2.755 \pm 0.017 \mathrm{~K}$. Although the significance of such large $\chi_{\min }^{2}$ should not be overemphasized in view of the possibility of unrecognized systematic errors, it may be noted that there is a tendency for the brightness temperatures to be slightly lower at $\mathrm{cm}$ than at $\mathrm{mm}$ wavelengths. A Bose-Einstein spectrum scores somewhat better. If $\hat{\mathbf{\Omega}}_{b}=0.1$, we find $\mu_{0}=(4.1 \pm 1.7) \times 10^{-3}, T_{0}=2.793 \pm 0.019 \mathrm{~K}$, with $\chi_{\min }^{2}=11.8$ for 10 degrees of freedom. The minimum $\chi^{2}$ is slightly larger both for $\hat{\Omega}_{b}=1\left[\chi_{\min }^{2}=12.4, \mu_{0}=(1.08 \pm 0.44) \times 10^{-2}\right]$ and for $\Omega_{b}=0.01\left[\chi_{\min }^{2}=12.4, \mu_{0}=(3.4 \pm 1.5) \times 10^{-3}\right]$. (The results are insensitive to the value of $\hat{\Omega}$, as a 
consequence of the fact that Bose-Einstein distortions arise in the radiation-dominated era.) $\mathrm{A}$ nonzero chemical potential is suggested at the $\approx 2.4 \sigma$ level.

\section{ISOTROPY}

Several excellent general studies on the angular distribution of the MWBR can be found in the recent literature. The comprehensive review by Partridge (1988) includes a careful discussion of observational problems. Anisotropies to be expected in the framework of different cosmological scenarios have been skillfully reviewed by Hogan, Kaiser and Rees (1982), Kaiser and Silk (1986), Efstathiou (1986), and Bond (1988). We will recall here only a few basic points.

\subsection{The Data}

The results of the Relict experiment, which has provided a high-sensitivity map of the whole sky, are described by Strukov and Skulachev (1988). We refer to his contribution for a discussion of the dipole anisotropy and of constraints on higher order multipoles.

TABLE 2. Recent Measurements of Anisotropies on Intermediate and Small Angular Scales

\begin{tabular}{|c|c|c|c|}
\hline Reference & $\lambda(\mathrm{cm})$ & $\theta$ & $\Delta T / T$ \\
\hline Davies et al. (1987) & 3 & $8^{\circ}$ & $3.7 \times 10^{-5}$ \\
\hline Melchiorri et al. (1981) & 0.07 & $6^{\circ}$ & $4 \times 10^{-5}$ \\
\hline Watson et al. (1989) & 3 & $5.6^{\circ}$ & $\leq 3 \times 10^{-5}$ \\
\hline Mandolesi et al. (1986a) & 3 & $2^{\circ}-6^{\circ}$ & $\leq 5-7 \times 10^{-4}$ \\
\hline Dall'Oglio and de Bernardis (1988) & 0.2 & $1.3^{\circ}$ & $\leq 2.5 \times 10^{-4}$ \\
\hline Uson and Wilkinson (1985) & 1.5 & 4.5 & $\leq 5 \times 10^{-5 t}$ \\
\hline Readhead et al. (1989) & 1.5 & $2^{\prime}$ & $\leq 1.5 \times 10^{-5}$ \\
\hline Martin and Partridge (1988) & 6 & $36^{\prime \prime}-160^{\prime \prime}$ & $(1.3 \pm 0.2) \times 10^{-4}$ \\
\hline Martin and Partridge (1988) & 6 & $18^{\prime \prime}-80^{\prime \prime}$ & $(1.7 \pm 0.5) \times 10^{-4}$ \\
\hline Fomalont et al. (1988) & 6 & $60^{\prime \prime}$ & $\leq 0.6 \times 10^{-4}$ \\
\hline Fomalont et al. (1988) & 6 & $30 "$ & $\leq 0.8 \times 10^{-4}$ \\
\hline Fomalont et al. (1988) & 6 & $18 "$ & $\leq 1.2 \times 10^{-4}$ \\
\hline Hogan and Partridge (1989) & 2 & $18^{\prime \prime}$ & $\leq 1.6 \times 10^{-4}$ \\
\hline Fomalont et al. (1988) & 6 & $12^{\prime \prime}$ & $\leq 8.5 \times 10^{-4}$ \\
\hline Kreysa and Chini (1989) & 0.13 & $11^{\prime \prime}$ & $\leq 2.6 \times 10^{-4}$ \\
\hline Hogan and Partridge (1989) & 2 & $5.3^{\prime \prime}$ & $\leq 6.3 \times 10^{-4}$ \\
\hline
\end{tabular}

$\dagger$ We quote here and in Figure 1 the 95\% upper limit obtained by Lasenby and Kaiser (1989) from a maximum likelihood analysis of Uson and Wilkinson's data; the originally reported limit was lower by a factor $\approx 2$.

Recent results on intermediate and small angular scales are listed in Table 2 and displayed in Figure 1. Significant fluctuations with an observed standard deviation of $3.7 \times 10^{-5}$ on scales of $8^{\circ}-10^{\circ}$ have been reported by the Jodrell-IAC group (Davies et al. 1987), at $\lambda=2.87 \mathrm{~cm}$. While recognizing that structure in the radio continuum emission from our Galaxy may contribute appreciably, the authors argue that a substantial part of the signal is probably intrinsic. Fluctuations of similar amplitude on an angular scale of $6^{\circ}$ were previously observed by Melchiorri et al. (1981) at submillimeter wavelengths; again, the interpretation of the result depends on an uncertain correction for the galactic contribution. More recently, the JodrellIAC group (Watson et al. 1989) has obtained new data on an angular scale $\cong 5^{\circ}$. They were able to identify all features in the scan that have temperature excesses $\Delta T / T>3 \times 10^{-5}$ with beam-diluted known sources, with the exception of a peak in the direction $\alpha=14^{h} 40^{m}, \delta \sim 40^{\circ}$ (see also Vittorio et al. 1989). The Relict experiment lacked sufficient sensitivity to test this result directly; it provides, however, strong constraints on any spectrum of primordial perturbations that can account for it (Klypin 1989, personal communication). 


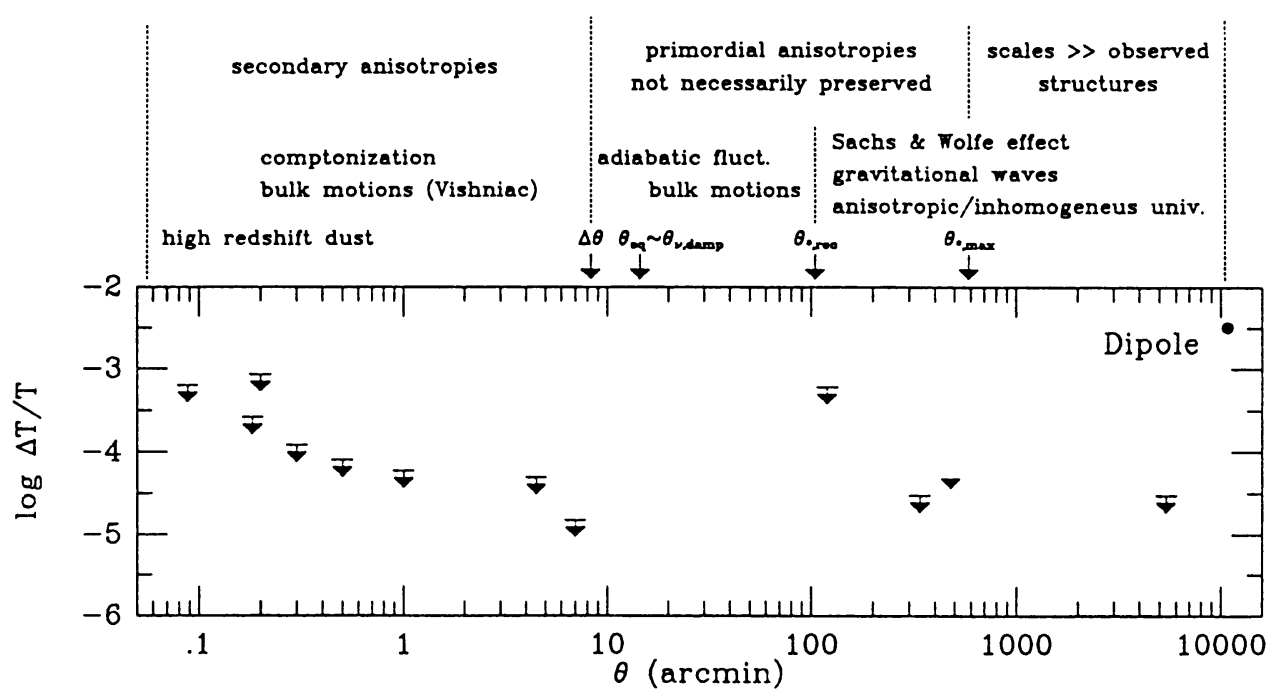

Figure 1. Recent measurements of MWBR anisotropies. Representative values for some characteristic angular scales $(\Omega=1)$ and an (incomplete) summary of mechanisms that may produce anisotropies are also shown.

High-sensitivity observations by several groups have led to remarkably tight upper limits on arcminute scales. On still smaller angular scales, around or below 1', a signal has definitely been detected. Its most likely interpretation, however, is confusion noise due to discrete sources below the detection limit (Fomalont et al. 1988; Franceschini et al. 1989; Hogan and Partridge 1989).

Sunyaev-Zeldovich (1972) dips on scales of a few arcminutes, in the directions of three rich clusters of galaxies, were the first small-scale anisotropies for which detection at a high level of significance has been reported (Birkinshaw 1989, and references therein). On the other hand, many years of experimental work have revealed several astrophysical and instrumental effects that may distort the results (cf. Partridge et al. 1987); the rich astrophysical information that can be provided by these measurements certainly deserves further observational effort.

\subsection{Theoretical Framework}

2.2.1. Characteristic scales. For simplicity we confine ourselves to $\Omega^{-1} \ll z \ll z_{e q}$ ( $z_{e q}$ being the redshift of equal matter and radiation densities). Then the angle subtended by a comoving length $L_{0}$ is essentially independent of $z$ :

$$
\theta=8.33 \times 10^{-3} \frac{L_{0}}{100 \mathrm{Mpc}} \frac{H_{0}}{50} \Omega \mathrm{rad} \simeq 0.477 \frac{L_{0}}{100 \mathrm{Mpc}} \frac{H_{0}}{50} \Omega .
$$

The region that came within the horizon at the redshift of last scattering, $z_{*}$, subtends an angle $\theta_{*} \approx\left(\Omega / z_{*}\right)^{1 / 2}$, which may range from $\theta_{*, e c} \simeq 1^{\circ} .75 \Omega^{1 / 2}$ (last scattering at the canonical epoch of recombination) to $\theta_{*, \max }=16^{\circ} .3\left(H_{0} / 50\right)^{1 / 3} \Omega_{b}^{1 / 3} \Omega^{1 / 3}$, in the case of the latest possible $z_{*}$. The interpretation of isotropy measurements on scales larger than $\approx 10^{\circ}$ is then independent of the uncertain ionization history of the universe for $z<1000$. On the other hand, the corresponding comoving length is of the order of few $\times 1000 \mathrm{Mpc}$; hence inferences on the origin of known 
structures would critically depend on the assumed extrapolation of the density fluctuation spectrum to much smaller scales.

A second important scale is that corresponding to the thickness of the last scattering surface, due to the finite time required for recombination of the primeval plasma. For a standard recombination, the comoving width of the last scattering shell is $\Delta L=14 \hat{\Omega}^{-1 / 2} \mathrm{Mpc}$ and the corresponding angular size is $\Delta \theta=8: 3 \Omega^{-1 / 2}$. Perturbations on scales smaller than $\Delta L$ become transparent when $\tau \gg 1$ (the optical depth of the universe increases very rapidly with increasing $z$ in the relevant range: $\tau(z) \simeq 0.37(z / 1000)^{14.25}$ [Jones and Wyse 1985]), and the corresponding temperature fluctuations are drastically smoothed out.

Even MWBR anisotropies on scales larger than $\Delta L$ (but smaller than $L_{*}$ ) do not necessarily survive. A modest amount of reheating may suffice to yield $\tau>1$ at redshifts well below 1000. In this case the universe becomes transparent much more gradually, and the effective width of the last scattering shell is of the order of the size of the particle horizon at $z_{*}$. A strong (exponential) damping of primeval anisotropies occurs only if re-ionization takes place at redshifts $\approx \mathrm{few} \times z_{*}$. Such early re-ionization seems unlikely in cold dark matter-dominated models, but it is expected in the isocurvature baryon models (Peebles 1989).

The proper radius of curvature of the hypersurface $t=$ const in the Friedman-RobertsonWalker metric subtends an angle (Peebles 1980):

$$
\theta_{c} \approx 0.5 \Omega(1-\Omega)^{-1 / 2} \mathrm{rad} \text {. }
$$

The spectrum of primordial density fluctuations is also expected to have several characteristic scales imprinted in it (see, e.g., Silk 1984). Fluctuations on comoving scales larger than the horizon at the equivalence epoch $\left(L_{e q}=50 \hat{\Omega}^{-1} \mathrm{Mpc}\right)$ grow uninterruptedly, while the growth of smaller scales is suppressed between the time they cross the horizon and $z_{\text {eq. }}$. Adiabatic fluctuations in a baryon-dominated universe are damped by radiative diffusion and viscosity on comoving scales below $L_{\text {damp }} \approx 6.5 \hat{\Omega}^{-5 / 6} \mathrm{Mpc}$. In a universe dominated by hot dark matter particles, such as massive neutrinos, with mass $m_{v}=25 \hat{\Omega} \mathrm{eV}$, free streaming erases density perturbations on scales smaller than the horizon at the moment when these particles ceased to be relativistic: $L_{v \text {,damp }}=52 \hat{\Omega}^{-1} \mathrm{Mpc}$. Density fluctuations in the range $L_{\text {damp }}<L<L_{\text {, rec }}$, with $L_{*, \text { rec }}=370 \hat{\Omega}^{-1 / 2} \mathrm{Mpc}$ the horizon at recombination in comoving coordinates, are amplified by a velocity overshoot effect. The corresponding angular scales are $\theta_{* \text {,rec }}=1^{\circ} .75 \Omega^{1 / 2}$, $\theta_{e q} \simeq 14^{\prime}: 3\left(H_{0} / 50\right)^{-1}, \theta_{v, d a m p} \simeq 15^{\prime}\left(H_{0} / 50\right)^{-1}, \theta_{\text {damp }} \simeq 1^{\prime} .9 \Omega^{1 / 6}\left(H_{0} / 50\right)^{-2 / 3}$.

2.2.2. Mechanisms producing anisotropies on intermediate and large scales. The dominant source of anisotropy on scales larger than $\theta_{*}$ but excluding the dipole are fluctuations $\delta \phi$ of the gravitational potential (Sachs and Wolfe 1967; Traschen and Eardley 1986, and references therein). The amplitude of the corresponding temperature fluctuation is about equal to the density contrast in the matter, $\delta=(\delta \rho / \rho)$, when the perturbation appears on the horizon, but the signs are opposite (Peebles 1980): $\Delta T / T \approx-\delta\left(L / L_{H}\right)^{2}=-\delta_{L=L_{H}}$. In open universes, this equation holds only for $\theta<\theta_{c}$ (equation [2]); for larger scales the gravitational deceleration is unimportant, and small density fluctuations stop growing at $z \approx \Omega^{-1}$. For spherical lumps, Kaiser (1982) has found that the r.m.s. temperature fluctuations are roughly equal to their present density contrast: $\left\langle(\Delta T / T)^{2}\right\rangle^{1 / 2} \approx \delta_{0}$.

Dynamic inhomogeneities between the observer and his last scattering surface induce second-order Sachs and Wolfe (1967) anisotropies (Rees and Sciama 1968). The profile of the temperature perturbations is, in general, rather complex. In the linear regime the magnitude of the perturbation is (Kaiser 1982): $\left\langle(\Delta T / T)^{2}\right\rangle^{1 / 2} \approx \delta\left(L / L_{H}\right)^{3}$. The effect of multiple inhomogeneities along the line of sight, however, is additive; hence, as shown by Dyer and Ip (1988), it is possible to construct self-consistent models whereby these second-order anisotropies are larger than those produced at the last scattering surface. Such models generally require lumps with very large masses, of the order of $10^{19} M_{\odot}$, and a slow growth rate of the density 
contrasts.

MWBR temperature profiles in representative inhomogeneous and anisotropic cosmological models have been investigated by Barrow, Juszkiewicz and Sonoda (1983), Lukash and Novikov (1985), and Bajtlik et al. (1986) and references therein. If $\Omega=1$, a pure quadrupole harmonic is generated. If $\Omega<1$ in homogeneous anisotropic cosmologies, hot spots may appear (although this is not a general property of open models), as first noticed by Novikov (1968) with a typical angular size $\approx 80^{\circ} \Omega$; in inhomogeneous universes a dipole appears, and, as $\Omega$ decreases, the relative importance of the quadrupole falls, while there is a build-up of power at harmonics with $l$-number $\approx \pi / \Omega$.

In addition to the scalar modes discussed so far, temperature fluctuations may arise from vector and tensor modes. Vector modes are determined by the rotation (vorticity) tensor which, in the linear theory, is not coupled to the density fluctuations (Sachs and Wolfe 1967); constraints on cosmological turbulence from the MWBR isotropy have been investigated by Kurskov and Ozernoi (1978) and Danese and De Zotti (1980). The anisotropies associated with tensor perturbations (gravitational waves) are proportional to the wave amplitudes and are expected to be particularly important on scales $=\theta_{* \text {,rec }}$, also because the wave amplitudes decline when the waves enter the horizon in the matter-dominated era (Starobinskii 1985, and references therein). Current limits on MWBR temperature fluctuations have allowed the setting of strong constraints on the energy density of any gravitational radiation background (Linder 1988a) as well as on physical processes that can generate it, such as a cosmological phase transition associated with primordial inflation and oscillating cosmic string loops (Linder 1988b); for example, the symmetry-breaking scale $M_{X}$ must be much smaller than the Planck mass $\left(M_{X}<7.5 \times 10^{-4} M_{p l}\right)$, i.e., the inflationary epoch cannot be too close to the Planck time.

2.2.3. Scales smaller than $\boldsymbol{\theta}_{*}$. If the photon entropy per baryon is constant (adiabatic or isentropic perturbations), temperature fluctuations of amplitude $\Delta T / T=(1 / 3)(\delta \rho / \rho)$ are present prior to recombination (Silk 1967). The amplitude of these temperature anisotropies, however, is reduced by the substantial optical depth of the universe when perturbations on scales $<\Delta L$ become transparent and by destructive interference of photons received from both peaks and troughs (Sunyaev and Zeldovich 1970). The peculiar velocity field associated with density perturbations gives an additional contribution of order $\Delta T / T \approx V / c \approx(\delta \rho / \rho)(L / c t)_{z=z_{z}}$, which dominates for scales close to (but smaller than) $L_{*}$. A precise calculation must take into account the fuzziness of the last scattering surface and the specific strength of anticorrelations between velocities of electrons in neighbouring regions.

If the fluctuations were initially perturbations in the entropy, with local conservation of energy (i.e., without perturbations in space curvature: isocurvature fluctuations), the expected small-scale anisotropies are similar to those arising from isentropic fluctuations, but, on larger scales, are about an order of magnitude larger (Suto, Sato and Kodama 1985; Efstathiou and Bond 1986).

Predictions of temperature fluctuations for a variety of models of structure formation have been summarized and compared with observational limits by Bond (1988). Bond and Efstathiou (1987; see also Vittorio and Juskiewicz 1987; Martinez-Gonzalez and Sanz 1989) have investigated in detail the texture of the MWBR using the theory of two-dimensional Gaussian random fields.

2.2.4. Cosmic strings. These linear topological defects, predicted by some grand unified theories to form during symmetry-breaking phase transitions in the very early universe, may provide a physical mechanism for producing large-scale structure. Anisotropies generated in the string scenario are inherently non-Gaussian and have the unique signature of producing steplike discontinuities in the intensity of the MWBR (Bouchet, Bennett and Stebbins 1988, and references therein). 


\subsection{Secondary Anisotropies}

As mentioned in secton 2.2.1, if the intergalactic medium is reionized, primeval temperature fluctuations on scales of up to $\approx 10^{\circ}$ may be erased. The new small-scale anisotropies due to Thomson scattering off electrons that are undergoing bulk motions induced by density perturbations are unobservably small in the linear theory, because the fuzziness of the last scattering shell causes contributions from crests and troughs of any plane wave to interfere destructively. However, as shown by Vishniac (1987), significant arcminute temperature fluctuations can arise as a second-order effect, proportional to $\delta n_{e} V$, where $\delta n_{e}$ is the inhomogeneity in the electron density and $V$ is the peculiar velocity.

Comptonization by inhomogeneously distributed hot gas (see Bond 1988 and references therein for a thorough discussion) is an important source of secondary anisotropies in scenarios involving large-scale cosmic explosions, decay of superconducting cosmic strings, and collapse and fragmentation of pancakes. In the cold dark matter scenario, the anisotropies induced by the hot gas component of bound structures are dominated by the rare large clusters of galaxies at moderate redshifts. Bond (1988) and Cole and Kaiser (1988) find $\Delta T / T \approx 10^{-5}$ on arcminute scales. Schaeffer and Silk's (1988) estimate is about one order of magnitude larger as a consequence of having assumed a much more extended distribution of hot gas.

The intensity fluctuations $\Delta I_{v} / I_{v}$ predicted by primeval dust models for the submillimeter excess are greater than or equal to a few percent on scales $\approx 10^{\prime \prime}$ (at $\lambda \leq 1 \mathrm{~mm}$ ) if the dust is clumped in normal spiral galaxies, but they can be much lower if the sources are very small and numerous. The Kreysa and Chini (1989) upper limit at $1.3 \mathrm{~mm}$ already sets interesting constraints on the space density of dust clouds and on their clustering properties (see Bond, Carr and Hogan 1989).

\subsection{Interpretation of Anisotropy Measurements}

The problems associated with the statistical procedures for deducing upper limits on temperature fluctuations have been highlighted by Kaiser and Silk (1986) and Lasenby and Kaiser (1989).

The comparison of experimental data with models is further complicated by astrophysical effects. As discussed by Scaramella and Vittorio (1989), the observable portion of the last scattering surface does not adequately sample very long density waves. Thus, measured largescale anisotropies may be significantly different from the theoretically computed expectation values. For example, the predicted distribution of quadrupole amplitudes has a standard deviation as large as $63 \%$. On small scales (below several arcminutes), multiple gravitational lensing generally leads to dilution of the anisotropy correlation function $C(\theta)$ (Linder 1989, and references therein). As a consequence, the observed temperature fluctuations on scales smaller than their intrinsic coherence angle can be substantially enhanced (recall that, for example, a double beam experiment with a beam throw $\theta$ measures $\langle\Delta T / T\rangle^{2}=2[C(0)-C(\theta)]$ ); on larger scales they are mildly attenuated.

\subsection{Fluctuations Due to Discrete Sources}

Small-scale temperature fluctuations due to the nonuniform space distribution of discrete sources may seriously hinder searches for primordial anisotropies. Only within a relatively narrow frequency range (from $\cong 20 \mathrm{GHz}$ to $\cong 200 \mathrm{GHz}$, where the relative intensity of local backgrounds is minimum: see Figure 2) is the contribution of sources to anisotropies on scale $<1^{\circ}$ expected to be $\Delta T / T \leq 10^{-5}$ (Franceschini et al. 1989).

On the other hand, fluctuations due to sources are themselves interesting quantities to measure. For example, they may be informative on the large-scale distribution of radio sources, which, in turn, may reflect the large-scale distribution of matter in general. As shown 


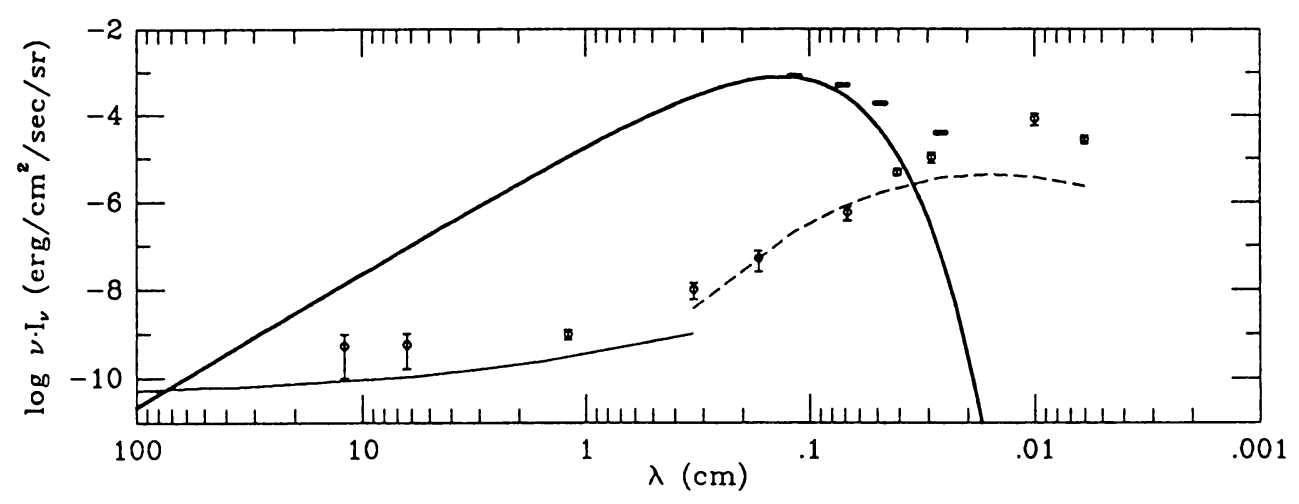

Figure 2. Comparison of the MWBR intensity $\left(T_{0}=2.74 \mathrm{~K}\right)$ with that of local backgrounds. The dashed line shows the summed contributions of non-evolving disk galaxies and evolving, actively starforming galaxies (Danese et al. 1987); the light solid line shows that of extragalactic radio sources; the data points are the observed emission from our own galaxy in the direction of the galactic pole (Sironi and Bonelli 1986; Mandolesi et al. 1986b; Fixen et al. 1983; Lubin et al. 1985; Halpern et al. 1988). The heavy bars display the results of Matsumoto et al. (1988).

by De Zotti and Toffolatti (1989), if radio sources have a power-law two-point correlation function, $\left[\xi(r)=\left(r / r_{o}\right)^{-1.8}\right]$, for $r_{0} \geq 30-40 \mathrm{Mpc}\left(H_{0}=50\right)$, the fluctuations are dominated by the effect of clustering. The upper limit set by Lasenby and Davies (1983) at $\lambda=5 \mathrm{~cm}$ already implies that $r_{0}$ cannot be much larger than $40 \mathrm{Mpc}$. This constraint is comparable to that obtained from the study of the two-point angular correlation function of sources in the MG II survey (Langston 1989).

Acknowledgments-G.D.Z. thanks A. Klypin, I. Strukov, and V. Lukash for useful discussions. We are indebted to R. Bond, B. Carr, G. Field, M. Halpern, S. Hayakawa, H. Gush, E. Palazzi, R.B. Partridge, Y. Rephaeli, R. Scaramella, and I. Strukov for having provided us with preprints and reprints of their recent papers. Work supported in part by MPI and CNR (through GNA and PSN).

\section{REFERENCES}

Bajtlik, S., Juszkiewicz, R., Proszynski, M., and Amsterdamski, P. 1986, Ap. J., 300, 463.

Barcons, X. 1987, Ap. J., 313, 547.

Barcons, X., and Fabian, A.C. 1988, M.N.R.A.S., 230, 139.

Barrow, J.D., Juszkiewicz, R., and Sonoda, D.H. 1983, Nature, 305, 397.

Bernstein, et al. 1989, Ap. J., Letters, 337, L1.

Bersanelli, M., et al. 1989, Ip. J., 339, 632.

Birkinshaw, M. 1989, in Proc. Workshop "Cosmic Microwave Background: 25 Years Later."

Bond, J.R. 1988, in The Early Universe, eds. W.G. Unruh and G.W. Semenoff, Dordrecht, Reidel, p. 283.

Bond, J.R., Carr, B.J., and Hogan, C.J. 1986, Ap. J., 306, 428.

Bond, J.R., Carr, B.J., and Hogan, C.J. 1989, Ap. J., submitted.

Bond, J.R., and Efstathiou, G. 1987, M.N.R.A.S., 226, 655.

Bouchet, F.R., Bennett, D.P., and Stebbins, A. 1988, Nature, 335, 410.

Burigana, C. 1989, thesis, University of Padua.

Carr, B.J. 1988, in Comets to Cosmology, Lecture Notes in Physics, 297, 265.

Cole, S., and Kaiser, N. 1988, M.N.R.A.S., 233, 637. 
Crane, P., et al. 1989, Proc. Moriond Astrophysics Meeting, March 1989 (Gif sur Yvette: Editions Frontières)

Dall'Oglio, G., and de Bernardis, P. 1988, Ap. J., 331, 547.

Danese, L., and De Zotti, G. 1977, Rivista Nuovo Cimento, 7, 277.

Danese, L., and De Zotti, G. 1980, Astr. Ap., 87, 303.

Danese, L., and De Zotti, G. 1981, Astr. Ap., 94, L33.

Danese, L., De Zotti, G., Franceschini, A., and Toffolatti, L. 1987, Ap. J. Letters, 318, L15.

Danese, L., and Partridge, R.B. 1989, Ap. J., 342, 604.

Davies, R.D., et al. 1987, Nature, 326, 462.

De Amici, G., et al. 1989, preprint.

De Amici, G., et al. 1988, Ap. J., 329, 556.

De Amici, G., Smoot, G.F., Friedman, S.D., and Witebsky, C. 1985, Ap. J., 298, 710.

De Zotti, G. 1986, in Prog. Part. Nucl. Phys., 17, ed. A. Faessler, 117.

De Zotti, G., and Toffolatti, L. 1989, in Highlights of Astronomy, 8, 681.

Dyer, C.C., and Ip, S.S. 1988, M.N.R.A.S., 235, 895.

Efstathiou, G. 1986, in Proc. 2nd ESO-CERN Symp. Cosmology, 103.

Efstathiou, G., and Bond, J.R. 1986, M.N.R.A.S., 218, 103.

Fabbri, R. 1981, Ap. Space Sci., 77, 529.

Fabbri, R., and Melchiorri, F. 1979, Astr. Ap., 78, 376.

Field, G.B., and Perrenod, S. 1977, Ap. J., 215, 717.

Field, G.B., and Walker, T.P. 1989, Phys. Rev. Letters, submitted.

Fixen, D.J., Cheng, E.S., and Wilkinson, D.T. 1983, Phys. Rev. Letters, 50, 620.

Fomalont, E.B., et al. 1988, A. J., 96, 1187.

Franceschini, A., Toffolatti, L., Danese, L., and De Zotti, G. 1989, Ap. J., in press.

Gould, R.J, and Rephaeli, Y. 1978, Ap. J., 219, 12.

Guilbert, P.W., and Fabian, A.C. 1986, M.N.R.A.S., 220, 439.

Gush, H.P. 1981, Phys. Rev. Letters, 47, 745.

Halpern, M., Benford, R., Meyer, S., Muehlner, D., and Weiss, R. 1988, Ap. J., 332, 596.

Hayakawa, S., et al. 1987, Publ. Astron. Soc. Japan, 39, 941.

Hogan, C.J., Kaiser, N., and Rees, M.J. 1982, Phil. Trans. R. Soc. Lond. A, 307, 97.

Hogan, C., and Partridge, R.B. 1989, Ap. J. Letters, 341, L29.

Johnson, D.G., and Wilkinson, D.T. 1987, Ap. J. Letters, 313, L1.

Jones, B.J.T., and Wyse, R.F.G. 1985, 149, 144.

Kaiser, N. 1982, M.N.R.A.S., 198, 1033.

Kaiser, N., and Silk, J. 1986, Nature, 324, 529.

Kawasaki, M., and Sato, K. 1987, Publ. Astr. Soc. Japan, 39, 837.

Kreysa, E., and Chini, A. 1989, in Proc. 3rd ESO/CERN, 433.

Kogut, A., et al. 1988, Ap. J., 325, 1.

Kurskov, A.A., and Ozernoi, L.M. 1978, Ap. Space Sci., 56, 67.

Lacey, C.G., and Field, G.B. 1988, Ap. J. Letters, 330, L1.

Langston, G. 1989, in Large-Scale Structure and Motions in the Universe, ed. M. Mezzetti, G. Giuricin, F. Mardirassian, and M. Ramella, Dordrecht, Kluwer Academic Publisher, 387.

Lasenby, A.N., and Davies, R.D. 1983, M.N.R.A.S., 203, 1137.

Lasenby, A.N., and Kaiser, N. 1989, preprint.

Levin, S.M., et al. 1988, Ap. J., 334, 14.

Linder, E.V. 1988a, Ap. J., 326, 517.

Linder, E.V. 1988b, Astr. Ap., 204, L11.

Linder, E.V. 1989, M.N.R.A.S., submitted.

Lubin, P. 1982, in Proc. International School of Physics, Course on Gamow Cosmology, Varenna.

Lubin, P., Villela, T., Epstein, G., and Smoot, G.F. 1985, Ap. J. Letters, 298, L1.

Lukash, V.N., and Novikov, I.D. 1985, Nature, 316, 46.

Mandolesi, N., et al. 1986a, Nature, 319, 751.

Mandolesi, N., et al. 1986b, Ap. J., 310, 561.

Martin, H.M., and Partridge, R.B. 1988, Ap. J., 324, 794.

Martinez-Gonzalez, E., and Sanz, J.L. 1989, M.N.R.A.S., 237, 939.

Matsumoto, T., et al. 1988, Ap. J., 329, 567. 
McDowell, J.C. 1986, M.N.R.A.S., 223, 763.

Melchiorri, F., Melchiorri, B., Ceccarelli, C., and Pietranera, L. 1981, Ap. J. Letters, 250, L1.

Meyer, D.M., and Jura, M. 1985, Ap. J., 297, 119.

Meyer, D.M., Roth, K.C., and Hawkins, I. 1989, Ap. J. Letters, in press.

Negroponte, J. 1986, M.N.R.A.S., 222, 19.

Novikov, I.D. 1968, Astr. Zh., 45, 538 [Sov. Astr., 12, 427].

Partridge, R.B. 1988, Rep. Prog. Phys., 51, 647.

Partridge, R.B., Perley, R.A., Mandolesi, N., and Delpino, F. 1987, Ap. J., 317, 112.

Peebles, P.J.E. 1980, The Large-Scale Structure of the Universe (Princeton: Princeton University Press).

Peebles, P.J.E. 1989, in Large-Scale Structure and Motions in the Universe, 119.

Peterson, J.B., Richards, P.L., and Timusk, T. 1985, Phys. Rev. Letters, 55, 332.

Raffelt, G., Dearborn, D., and Silk, J. 1989, Ap. J., 336, 61.

Readhead, A.C.S., et al. 1989, Ap. J., submitted.

Rees, M.J., and Sciama, D.W. 1968, Nature, 217, 511.

Rephaeli, Y. 1980, Ap. J., 241, 858.

Richards, P.L. 1989, in Proc. Workshop "Cosmic Microwave Background: 25 Years Later."

Rogers, R., and Field, G.B. 1989, preprint.

Sachs, R.K., and Wolfe, A.M. 1967, Ap. J., 147, 73.

Scaramella, R., and Vittorio, N. 1989, preprint.

Schaeffer, R., and Silk, J. 1988, Ap. J., 333, 509.

Silk, J. 1967, Nature, 215, 1155.

Silk, J. 1984, in Proc. First ESO-CERN Symp., 225.

Sironi, G., and Bonelli, G. 1986, Ap. J., 311, 418.

Sironi, G., et al. 1989, Ap. J., submitted.

Smoot, G.F., Levin, S.M., Witebsky, C., De Amici, G., and Rephaeli, Y. 1988, Ap. J., 331, 653.

Starobinskii, A.A. 1985, Pis' ma Astr. Zh., 11, 323 [Sov. Astr. Letters, 11, 133].

Strukov, I. A., and Skulachev, D. P. 1988, Sov. Sci. Rev. E, Ap. Space Phys, 6, 145.

Sunyaev, R.A., and Zeldovich, Ya.B. 1970, Ap. Space Sci., 7, 3.

Sunyaev, R.A., and Zeldovich, Ya.B. 1972, Comm. Ap. Space Phys., 4, 173.

Sunyaev, R.A., and Zeldovich, Ya.B. 1980, Ann. Rev. Astr. Ap., 18, 537.

Suto, Y., Sato, K., and Kodama, H. 1985, Ap. J. Letters, 292, L1.

Taylor, G.B., and Wright, E.L. 1989, Ap. J., 339, 619.

Traschen, J., and Eardley, D.M. 1986, Phys. Rev. D, 34, 1665.

Uson, J.M., and Wilkinson, D.T. 1985, Nature, 312, 427.

Vishniac, E.T. 1987, Ap. J., 322, 597.

Vittorio, N., de Bernardis, P., Masi, S., and Scaramella, R. 1989, Ap. J., 341, 163.

Vittorio, N., and Juskiewicz, R. 1987, Ap. J. Letters, 314, L29.

Watson, R. A., et al. 1989, in Large-Scale Structure and Motions in the Universe, 133.

Weiss, R. 1980, Ann. Rev. Astr. Ap., 18, 489.

Wright, E.L. 1979, Ap. J., 232, 348.

Wright, E.L. 1983, in IAU Symposium 104, 113.

J. Peebles: I wonder if the spread in dust grain temperature in the submillimeter excess model is as serious as would be suggested by the spreads observed in low red shifts objects. At redshift $z \approx 30$, the lower band would be at $\approx 100 \mathrm{~K}$, which would considerably squeeze the spread in temperatures.

G. De Zotti: This is a good question that deserves further investigation. The only detailed study that I know of (Bond, Carr and Hogan 1989) takes into account only the variation of dust temperature with redshift. In this case a relatively narrow dust emission spectrum is not too difficult to achieve: it is enough to make sure that the effective redshift range is not too large. In my view it is much more difficult to avoid the nonuniformities in the local radiation field heating the dust, and the differences in the far-infrared emission efficiency of grains of different sizes, i.e., the two effects that account for the broad far-infrared emission spectra of nearby sources. The analysis of Bond et al. indicates that the effect of the presence of the microwave background radiation is not large at submillimeter wavelengths, and temperatures of a few times $100 \mathrm{~K}$ do not seem particularly implausible. 